PROCEEDINGS OF THE

AMERICAN MATHEMATICAL SOCIETY

Volume 137, Number 3, March 2009, Pages 825-833

S 0002-9939(08)09541-5

Article electronically published on September 12, 2008

\title{
ARITHMETIC PROPERTIES OF NON-HARMONIC WEAK MAASS FORMS
}

\author{
KATHRIN BRINGMANN AND DAVID PENNISTON
}

(Communicated by Ken Ono)

\begin{abstract}
We prove the existence of an infinite family of non-harmonic weak Maass forms of varying weights and Laplace eigenvalues having algebraic coefficients, and show that the coefficients of these forms satisfy congruences of Ramanujan type.
\end{abstract}

\section{InTRODUCTION AND STATEMENT OF RESULTS}

A partition of a positive integer $n$ is a non-increasing sequence of positive integers whose sum is $n$. Let $p(n)$ denote the partition function, i.e., the number of partitions of $n$, and set $p(0):=1$. The generating function for $p(n)$ is given by

$$
P(q):=\sum_{n=0}^{\infty} p(n) q^{n}=\prod_{n=1}^{\infty} \frac{1}{1-q^{n}}=1+\sum_{n=1}^{\infty} \frac{q^{n^{2}}}{(1-q)^{2}\left(1-q^{2}\right)^{2} \cdots\left(1-q^{n}\right)^{2}}
$$

The arithmetic behavior of the partition function has been of great interest. For example, we have the famous Ramanujan congruences

$$
\begin{aligned}
p(5 n+4) & \equiv 0 \quad(\bmod 5), \\
p(7 n+5) & \equiv 0 \quad(\bmod 7), \\
p(11 n+6) & \equiv 0 \quad(\bmod 11)
\end{aligned}
$$

for every $n \geq 0$. In a celebrated paper Ono 15 treated this type of congruence systematically, proving that for any prime $\ell \geq 5$ there exist infinitely many nonnested arithmetic progressions $A n+B$ such that for every $n \geq 0$,

$$
p(A n+B) \equiv 0 \quad(\bmod \ell) .
$$

Now consider the function

$$
f(q):=1+\sum_{n=1}^{\infty} \frac{q^{n^{2}}}{(1+q)^{2}\left(1+q^{2}\right)^{2} \cdots\left(1+q^{n}\right)^{2}},
$$

which is one of the mock theta functions Ramanujan [16 defined in his last letter to Hardy in 1920. While $f(q)$ and $P(q)$ have similar shapes, $P(q)$ is (up to a power of $q)$ a modular form and $f(q)$ is not. However, $f(q)$ does constitute the "holomorphic part" of a weak Maass form (see Section 2 for the definition of weak Maass form). The behavior of many arithmetic functions (Hurwitz class numbers, Dyson's ranks, and coefficients of other mock theta functions, to name a few) is governed by the

Received by the editors July 23, 2007, and, in revised form, February 26, 2008.

2000 Mathematics Subject Classification. Primary 11F33.

(C) 2008 American Mathematical Society 825

Reverts to public domain 28 years from publication 
coefficients of weak Maass forms (see [2, 3, 4, 5, 6, 7, 8, 9, 10, 11, 12, 13, 14). For example, Ono and the first author used weak Maass forms [7] to show that Dyson's rank generating functions satisfy congruences of Ramanujan type.

Weak Maass forms have expansions involving Whittaker functions, and as in the theory of modular forms one is interested in forms whose expansions have algebraic coefficients. The known examples of such forms are harmonic, i.e., have Laplace eigenvalue zero. Here we show the existence of an infinite family of non-harmonic weak Maass forms of varying weights and Laplace eigenvalues possessing algebraic coefficients. In particular, suppose $b \geq 4$ is even, let $w$ be a nonnegative integer, let $\chi$ be a nontrivial Dirichlet character of conductor $b$ with $\chi(-1)=(-1)^{w+1}$, and write $z=x+i y$ with $x, y \in \mathbb{R}$. In Section 3 we explicitly construct a function $f_{w+\frac{1}{2}, \chi}(z)$ that has the shape

$$
f_{w+\frac{1}{2}, \chi}(z)=f_{w+\frac{1}{2}, \chi}^{h}(z)+f_{w+\frac{1}{2}, \chi}^{n h}(z)
$$

where

$$
\begin{aligned}
f_{w+\frac{1}{2}, \chi}^{h}(z):= & \widetilde{a}_{w+\frac{1}{2}}\left(-n_{0}\right) \widetilde{\mathcal{W}}_{\frac{3}{4}}^{w+\frac{1}{2}}\left(-4 \pi n_{0} y\right) e^{-2 \pi i n_{0} x} \\
& +\sum_{n>0} a_{w+\frac{1}{2}}(n) \mathcal{W}_{\frac{3}{4}}^{w+\frac{1}{2}}(4 \pi n y) e^{2 \pi i n x}, \\
f_{w+\frac{1}{2}, \chi}^{n h}(z):= & \sum_{n>0} a_{w+\frac{1}{2}}\left(-d n^{2}\right) \mathcal{W}_{\frac{3}{4}}^{w+\frac{1}{2}}\left(-4 \pi d n^{2} y\right) e^{-2 \pi i d n^{2} x} .
\end{aligned}
$$

Here $d$ and $n_{0}$ are positive integers and the functions $\mathcal{W}_{\frac{3}{4}}^{w+\frac{1}{2}}(y)$ and $\widetilde{\mathcal{W}}_{\frac{3}{4}}^{w+\frac{1}{2}}(y)$ are certain modified Whittaker functions defined in Section 2 , We prove the following result.

Theorem 1.1. Suppose $b \geq 4$ is even. Let $w$ be a nonnegative integer and let $\chi$ be a nontrivial Dirichlet character of conductor $b$ which is even (resp. odd) if $w$ is odd (resp. even). Then the function $f_{w+\frac{1}{2}, \chi}(z)$ is a weak Maass form of weight $w+\frac{1}{2}$ on $\Gamma_{0}\left(4 b^{2}\right)$ with Nebentypus $\chi$ and Laplace eigenvalue $\frac{1}{4} w(w-1)$, and the coefficients of $f_{w+\frac{1}{2}, \chi}^{h}(z)$ lie in the ring of integers $\mathcal{O}_{K}$ of some number field $K$.

The forms $f_{w+\frac{1}{2}, \chi}(z)$ are examples of weak Maass forms which we call good (see Section 2 for the definition). In particular, if we denote the incomplete gamma function by

$$
\Gamma(a, y):=\int_{y}^{\infty} e^{-t} t^{a-1} d t
$$

and $v:=w+\frac{1}{2} \in\left\{\frac{1}{2}, \frac{3}{2}\right\}$, then it follows from our construction that

$$
\begin{aligned}
f_{v, \chi}^{h}(z) & =\widetilde{a}_{v}\left(-n_{0}\right) q^{-n_{0}}+\sum_{n>0} a_{v}(n) q^{n}, \\
f_{v, \chi}^{n h}(z) & =\sum_{n>0} a_{v}\left(-d n^{2}\right) \Gamma\left(\frac{1}{2}, 4 \pi d n^{2} y\right) q^{-d n^{2}},
\end{aligned}
$$

where $q:=e^{2 \pi i z}$. Note that

$$
\frac{\partial}{\partial \bar{z}}\left(f_{v, \chi}^{h}(z)\right)=0
$$


and moreover one can show that

$$
\frac{\partial}{\partial \bar{z}}\left(f_{v, \chi}^{n h}(z)\right) \neq 0
$$

For this reason we call $f_{v, \chi}^{h}(z)$ the "holomorphic part" of $f_{v, \chi}(z)$ and $f_{v, \chi}^{n h}(z)$ the "nonholomorphic part" of $f_{v, \chi}(z)$.

Remark. It follows from our construction of $f_{v, \chi}(z)$ that there is some constant multiple of $f_{v, \chi}^{n h}(z)$ whose coefficients lie in the ring of integers of a number field $K$.

We also show that the coefficients of the $f_{w+\frac{1}{2}, \chi}^{h}(z)$ components of our nonharmonic weak Maass forms satisfy congruences of Ramanujan type.

Theorem 1.2. Let $f_{w+\frac{1}{2}, \chi}(z)$ be the weak Maass form of weight $w+\frac{1}{2}$ and Nebentypus $\chi$ constructed in the proof of Theorem 1.1, and decompose $f_{w+\frac{1}{2}, \chi}(z)$ as in (1.2). Suppose $\ell \nmid 6 b$ is prime, $p \nmid \ell d$ is an odd prime and $j \geq 1$. Then there exists an integer $m \geq 1$ such that for a positive proportion of the primes $Q$,

$$
a_{w+\frac{1}{2}}\left(Q^{3} \ell^{m} n\right) \equiv 0 \quad\left(\bmod \ell^{j}\right)
$$

for all $n>0$ with $(n, \ell Q)=1$ and $\left(\frac{-Q^{3} \ell^{m} d n}{p}\right)=-1$.

Two remarks.

1) From our construction of $f_{w+\frac{1}{2}, \chi}(z)$ it follows trivially that

$$
a_{w+\frac{1}{2}}(\ell n) \equiv 0 \quad\left(\bmod \ell^{r}\right)
$$

for $n>0$, where $r:=\left[\frac{w}{2}\right]$.

2) It seems likely that one can give distribution results on the coefficients of the forms $f_{w+\frac{1}{2}, \chi}^{h}(z)$ modulo $\ell^{j}$ as in [13], and asymptotics for these coefficients as in [2].

\section{General facts on Weak MaAss forms}

In this section we recall basic facts on weak Maass forms, which were first studied in [10. For $d$ odd, define $\epsilon_{d}$ by

$$
\epsilon_{d}:=\left\{\begin{array}{lll}
1 & \text { if } d \equiv 1 \quad(\bmod 4), \\
i & \text { if } d \equiv 3 \quad(\bmod 4) .
\end{array}\right.
$$

Suppose that $k \in \frac{1}{2}+\mathbb{Z}$, and let

$$
\Delta_{k}:=-4 y^{2} \frac{\partial^{2}}{\partial z \partial \bar{z}}+2 i k y \frac{\partial}{\partial \bar{z}}
$$

be the weight $k$ hyperbolic Laplacian, where $\frac{\partial}{\partial z}:=\frac{1}{2}\left(\frac{\partial}{\partial x}-i \frac{\partial}{\partial y}\right)$ and $\frac{\partial}{\partial \bar{z}}:=$ $\frac{1}{2}\left(\frac{\partial}{\partial x}+i \frac{\partial}{\partial y}\right)$.

Definition. Let $N$ be a positive integer, $\psi$ a Dirichlet character modulo $4 N$ and $g: \mathbb{H} \rightarrow \mathbb{C}$ a smooth function. We call $g(z)$ a weak Maass form of weight $k$ and Laplace eigenvalue $\lambda$ on $\Gamma_{0}(4 N)$ with Nebentypus $\psi$ if it satisfies the following three conditions: 
(1) For all $A=\left(\begin{array}{ll}a & b \\ c & d\end{array}\right) \in \Gamma_{0}(4 N)$ and all $z \in \mathbb{H}$, we have

$$
g(A z)=\psi(d)\left(\frac{c}{d}\right)^{2 k} \epsilon_{d}^{-2 k}(c z+d)^{k} g(z) .
$$

(2) We have $\Delta_{k} g=\lambda g$.

(3) The function $g(z)$ has at most linear exponential growth at all cusps. If $\lambda=0$, we say that $g(z)$ is harmonic.

Let us next recall the shape of the Fourier expansion of a weak Maass form. For this let $\nu, \mu \in \mathbb{C}$ and let $W_{\nu, \mu}(y)$ be the standard $W$-Whittaker function. Then $W_{\nu, \mu}(y)$ and $W_{-\nu, \mu}(-y)$ are linearly independent solutions of the differential equation

$$
\frac{\partial^{2} u}{\partial y^{2}}+\left(-\frac{1}{4}+\frac{\nu}{y}+\frac{\frac{1}{4}-\mu^{2}}{y^{2}}\right) u=0
$$

and can be distinguished by their asymptotic behavior, namely

$$
\left|W_{ \pm \nu, \mu}( \pm y)\right| \sim e^{\mp \frac{y}{2}}|y|^{ \pm \nu}
$$

as $|y| \rightarrow \infty$. For $y \in \mathbb{R} \backslash\{0\}$ define the functions

$$
\begin{aligned}
& \mathcal{W}_{s}^{k}(y):=|y|^{-\frac{k}{2}} W_{\operatorname{sgn}(y) \frac{k}{2}, s-\frac{1}{2}}(|y|), \\
& \widetilde{\mathcal{W}}_{s}^{k}(y):=|y|^{-\frac{k}{2}} W_{-\operatorname{sgn}(y) \frac{k}{2}, s-\frac{1}{2}}(-|y|) .
\end{aligned}
$$

A computation shows that $\mathcal{W}_{s}^{k}(y) e^{\frac{i x}{2}}$ and $\widetilde{\mathcal{W}}_{s}^{k}(y) e^{\frac{i x}{2}}$ are eigenfunctions of $\Delta_{k}$ with eigenvalue $s(1-s)+\frac{k^{2}-2 k}{4}$. Moreover, if $s \neq \frac{1}{2}$, then $y^{s-\frac{k}{2}}$ and $y^{1-s-\frac{k}{2}}$ are two linearly independent solutions of

$$
-y^{2} \frac{\partial^{2} u}{\partial y^{2}}-k y \frac{\partial u}{\partial y}=\left(s(1-s)+\frac{k^{2}-2 k}{4}\right) u .
$$

Using the translation invariance and properties (2) and (3) of a weak Maass form one can show that each weak Maass form $g(z)$ of weight $k$ and Laplace eigenvalue $\lambda$ has an expansion of the form

$$
\begin{aligned}
g(z)= & \sum_{\substack{n=-n_{0} \\
n \neq 0}}^{m_{0}} \widetilde{a}(n) \widetilde{\mathcal{W}}_{s}^{k}(4 \pi n y) e^{2 \pi i n x}+\sum_{n \in \mathbb{Z} \backslash\{0\}} a(n) \mathcal{W}_{s}^{k}(4 \pi n y) e^{2 \pi i n x} \\
& +a(0) y^{s-\frac{k}{2}}+\widetilde{a}(0) y^{1-s-\frac{k}{2}}
\end{aligned}
$$

where $s$ is a solution of

$$
s(1-s)+\frac{k^{2}-2 k}{4}=\lambda .
$$

Note that by (2.2), the first sum in (2.4) is responsible for the possible exponential growth of $g(z)$. We call a weak Maass form $g(z) \operatorname{good}$ if (1) $\widetilde{a}(n)=0$ for $n \geq 0$, and (2) there exists a positive integer $d$ such that $a(n) \neq 0$ implies that $n>0$ or $n=-d m^{2}$ for some nonzero integer $m$.

For functions $g: \mathbb{H} \rightarrow \mathbb{C}$ define the operator $R_{k}$ by

$$
R_{k}(g):=\frac{1}{2 \pi i} \frac{\partial g}{\partial z}-\frac{k}{4 \pi y} g .
$$

One can check (see [10] for the case of harmonic weak Maass forms) that this operator maps a weak Maass form of weight $k$ and Laplace eigenvalue $\lambda$ to a weak 
Maass form of weight $k+2$ and Laplace eigenvalue $\lambda+k$ with the same level and Nebentypus. We will refer to $R_{k}$ as the raising operator.

\section{Proof of Theorems 1.1 and 1.2}

We recall the following relations for Whittaker functions, which we will make use of in the proof of Theorem 1.1 (these follow, respectively, from 13.1.33 and 13.4.17, from 13.4.30, and from 13.4.31 and 13.4.33 of [1]).

$$
\begin{gathered}
W_{k, m}(y)=y^{\frac{1}{2}} W_{k-\frac{1}{2}, m-\frac{1}{2}}(y)+\left(\frac{1}{2}-k+m\right) W_{k-1, m}(y), \\
W_{k, m}(y)=y^{\frac{1}{2}} W_{k-\frac{1}{2}, m+\frac{1}{2}}(y)+\left(\frac{1}{2}-k-m\right) W_{k-1, m}(y), \\
y \frac{\partial}{\partial y}\left(W_{k, m}(y)\right)=\left(k-\frac{y}{2}\right) W_{k, m}(y)-\left[m^{2}-\left(k-\frac{1}{2}\right)^{2}\right] W_{k-1, m}(y) .
\end{gathered}
$$

Here and in the following, for all occurring square roots we take a branch of the logarithm with a cut which does not intersect the real axis.

Proof of Theorem 1.1. Assume that for each $v \in\left\{\frac{1}{2}, \frac{3}{2}\right\}$ we have a weight $v$ harmonic weak Maass form $f_{v, \chi}(z)$ on $\Gamma_{0}\left(4 b^{2}\right)$ with Nebentypus $\chi$ such that the Fourier coefficients of $f_{v, \chi}^{h}(z)$ lie in $\mathcal{O}_{K}$ (we delay the construction of the forms $f_{v, \chi}(z)$ to the end). Suppose that $w$ is even, and write $w=2 r$ (the case where $w$ is odd can be handled in a similar way). Beginning with $f_{\frac{1}{2}, \chi}(z)$, our proof proceeds by successively applying raising operators (2.6), yielding after $\ell$ steps $(0<\ell \leq r)$ a weight $2 \ell+\frac{1}{2}$ weak Maass form $f_{2 \ell+\frac{1}{2}, \chi}(z)$ on $\Gamma_{0}\left(4 b^{2}\right)$ with Nebentypus $\chi$ and Laplace eigenvalue $\frac{\ell(2 \ell-1)}{2}$. Computing the effect of the raising operators on Fourier expansions, we find that $f_{2 \ell+\frac{1}{2}, \chi}^{h}(z)$ has coefficients in $\mathcal{O}_{K}$ as well.

Define the functions $f_{2 \ell+\frac{1}{2}, \chi}(z)$ inductively by

$$
f_{2(\ell+1)+\frac{1}{2}, \chi}(z):=R_{2 \ell+\frac{1}{2}}\left(f_{2 \ell+\frac{1}{2}, \chi}(z)\right)
$$

for $0 \leq \ell<r$, and define the coefficients $a_{2 \ell+\frac{1}{2}}(n)$ and $\widetilde{a}_{2 \ell+\frac{1}{2}}(n)$ (for ease of notation we suppress the character $\chi$ ) for $0 \leq \ell \leq r$ (see (2.4) $)$ by

$$
\begin{aligned}
& f_{2 \ell+\frac{1}{2}, \chi}(z)=: \sum_{n \in \mathbb{Z} \backslash\{0\}} a_{2 \ell+\frac{1}{2}}(n) \mathcal{W}_{\frac{3}{4}}^{2 \ell+\frac{1}{2}}(4 \pi n y) e^{2 \pi i n x} \\
& +\sum_{\substack{n=-n_{0} \\
n \neq 0}}^{m_{0}} \widetilde{a}_{2 \ell+\frac{1}{2}}(n) \widetilde{\mathcal{W}}_{\frac{3}{4}}^{2 \ell+\frac{1}{2}}(4 \pi n y) e^{2 \pi i n x}+a_{2 \ell+\frac{1}{2}}(0)(4 \pi y)^{\frac{1}{2}-\ell}+\widetilde{a}_{2 \ell+\frac{1}{2}}(0)(4 \pi y)^{-\ell}
\end{aligned}
$$

(note that we have chosen the $s=\frac{3}{4}$ solution of (2.5)). We now compute the action of $R_{2 \ell+\frac{1}{2}}$ on the terms of this expansion. We show this computation only for the terms involving the functions $\mathcal{W}_{\frac{3}{4}}^{2 \ell+\frac{1}{2}}(y)$, as the others can be dealt with in a similar way.

Suppose $0 \leq \ell<r$. For the $n=0$ case we simply observe that

$$
R_{2 \ell+\frac{1}{2}}\left((4 \pi y)^{\frac{1}{2}-\ell}\right)=-(\ell+1)(4 \pi y)^{-\frac{1}{2}-\ell}
$$


and

$$
\left.R_{2 \ell+\frac{1}{2}}\left((4 \pi y)^{-\ell}\right)\right)=-\left(\ell+\frac{1}{2}\right)(4 \pi y)^{-1-\ell} .
$$

Next suppose $n<0$. We claim that

$$
R_{2 \ell+\frac{1}{2}}\left(\mathcal{W}_{\frac{3}{4}}^{2 \ell+\frac{1}{2}}(4 \pi n y) e^{2 \pi i n x}\right)=n\left(\ell^{2}+\frac{3 \ell}{2}+\frac{1}{2}\right) \mathcal{W}_{\frac{3}{4}}^{2 \ell+\frac{5}{2}}(4 \pi n y) e^{2 \pi i n x} .
$$

To see this, recall that for $y>0$,

$$
\mathcal{W}_{\frac{3}{4}}^{2 \ell+\frac{1}{2}}(-y)=y^{-\ell-\frac{1}{4}} W_{-\ell-\frac{1}{4}, \frac{1}{4}}(y) .
$$

Using (3.3) we find that

$$
\begin{aligned}
\frac{\partial}{\partial y}\left(W_{-\ell-\frac{1}{4}, \frac{1}{4}}(y)\right)=\frac{1}{y} & \left(-\ell-\frac{1}{4}-\frac{y}{2}\right) W_{-\ell-\frac{1}{4}, \frac{1}{4}}(y) \\
& +\frac{1}{y}\left(\ell^{2}+\frac{3 \ell}{2}+\frac{1}{2}\right) W_{-\ell-\frac{5}{4}, \frac{1}{4}}(y),
\end{aligned}
$$

and therefore

$$
\begin{aligned}
\frac{\partial}{\partial y}\left(\mathcal{W}_{\frac{3}{4}}^{2 \ell+\frac{1}{2}}(-y)\right) & =\left(-2 \ell-\frac{1}{2}\right) y^{-\ell-\frac{5}{4}} W_{-\ell-\frac{1}{4}, \frac{1}{4}}(y) \\
& -\frac{1}{2} y^{-\ell-\frac{1}{4}} W_{-\ell-\frac{1}{4}, \frac{1}{4}}(y)+\left(\ell^{2}+\frac{3 \ell}{2}+\frac{1}{2}\right) y^{-\ell-\frac{5}{4}} W_{-\ell-\frac{5}{4}, \frac{1}{4}}(y) .
\end{aligned}
$$

It follows that

$$
\begin{aligned}
\frac{\partial}{\partial z}\left(\mathcal{W}_{\frac{3}{4}}^{2 \ell+\frac{1}{2}}(-y) e^{-\frac{i x}{2}}\right)=i\left(\ell+\frac{1}{4}\right) & y^{-\ell-\frac{5}{4}} W_{-\ell-\frac{1}{4}, \frac{1}{4}}(y) e^{-\frac{i x}{2}} \\
& -\frac{i}{2}\left(\ell^{2}+\frac{3 \ell}{2}+\frac{1}{2}\right) y^{-\ell-\frac{5}{4}} W_{-\ell-\frac{5}{4}, \frac{1}{4}}(y) e^{-\frac{i x}{2}},
\end{aligned}
$$

and hence

$$
\begin{aligned}
R_{2 \ell+\frac{1}{2}}\left(\mathcal{W}_{\frac{3}{4}}^{2 \ell+\frac{1}{2}}(4 \pi n y) e^{2 \pi i n x}\right)= & n\left(\ell^{2}+\frac{3 \ell}{2}+\frac{1}{2}\right) \\
& \cdot(4 \pi|n| y)^{-\ell-\frac{5}{4}} W_{-\ell-\frac{5}{4}, \frac{1}{4}}(4 \pi|n| y) e^{2 \pi i n x},
\end{aligned}
$$

which is (3.4).

Now suppose $n>0$. Here the claim is that

$$
R_{2 \ell+\frac{1}{2}}\left(\mathcal{W}_{\frac{3}{4}}^{2 \ell+\frac{1}{2}}(4 \pi n y) e^{2 \pi i n x}\right)=n \mathcal{W}_{\frac{3}{4}}^{2 \ell+\frac{5}{2}}(4 \pi n y) e^{2 \pi i n x} .
$$

To see this, begin by noting that for $y>0$,

$$
\mathcal{W}_{\frac{3}{4}}^{2 \ell+\frac{1}{2}}(y)=y^{-\ell-\frac{1}{4}} W_{\ell+\frac{1}{4}, \frac{1}{4}}(y) .
$$

Using (3.3) we obtain

$$
\frac{\partial}{\partial y}\left(W_{\ell+\frac{1}{4}, \frac{1}{4}}(y)\right)=\frac{1}{y}\left(\ell+\frac{1}{4}-\frac{y}{2}\right) W_{\ell+\frac{1}{4}, \frac{1}{4}}(y)+\frac{1}{y}\left(\ell^{2}-\frac{\ell}{2}\right) W_{\ell-\frac{3}{4}, \frac{1}{4}}(y),
$$

and thus

$$
\frac{\partial}{\partial y}\left(\mathcal{W}_{\frac{3}{4}}^{2 \ell+\frac{1}{2}}(y)\right)=-\frac{1}{2} y^{-\ell-\frac{1}{4}} W_{\ell+\frac{1}{4}, \frac{1}{4}}(y)+\left(\ell^{2}-\frac{\ell}{2}\right) y^{-\ell-\frac{5}{4}} W_{\ell-\frac{3}{4}, \frac{1}{4}}(y) .
$$


Hence we find that

$\frac{\partial}{\partial z}\left(\mathcal{W}_{\frac{3}{4}}^{2 \ell+\frac{1}{2}}(y) e^{\frac{i x}{2}}\right)=\frac{i}{2} y^{-\ell-\frac{1}{4}} W_{\ell+\frac{1}{4}, \frac{1}{4}}(y) e^{\frac{i x}{2}}-\frac{i}{2}\left(\ell^{2}-\frac{\ell}{2}\right) y^{-\ell-\frac{5}{4}} W_{\ell-\frac{3}{4}, \frac{1}{4}}(y) e^{\frac{i x}{2}}$,

which gives

$$
\begin{aligned}
R_{2 \ell+\frac{1}{2}} & \left(\mathcal{W}_{\frac{3}{4}}^{2 \ell+\frac{1}{2}}(4 \pi n y) e^{2 \pi i n x}\right)=n(4 \pi n y)^{-\ell-\frac{1}{4}} W_{\ell+\frac{1}{4}, \frac{1}{4}}(4 \pi n y) e^{2 \pi i n x} \\
& -n\left(\ell^{2}-\frac{\ell}{2}\right)(4 \pi n y)^{-\ell-\frac{5}{4}} W_{\ell-\frac{3}{4}, \frac{1}{4}}(4 \pi n y) e^{2 \pi i n x} \\
& -n\left(2 \ell+\frac{1}{2}\right)(4 \pi n y)^{-\ell-\frac{5}{4}} W_{\ell+\frac{1}{4}, \frac{1}{4}}(4 \pi n y) e^{2 \pi i n x} .
\end{aligned}
$$

Now, equations (3.1) and (3.2) yield

$$
\begin{aligned}
W_{\ell+\frac{5}{4}, \frac{1}{4}}(y) & =y^{\frac{1}{2}} W_{\ell+\frac{3}{4}, \frac{3}{4}}(y)+(-\ell-1) W_{\ell+\frac{1}{4}, \frac{1}{4}}(y), \\
W_{\ell+\frac{3}{4}, \frac{3}{4}}(y) & =y^{\frac{1}{2}} W_{\ell+\frac{1}{4}, \frac{1}{4}}(y)+\left(\frac{1}{2}-\ell\right) W_{\ell-\frac{1}{4}, \frac{3}{4}}(y), \\
y^{\frac{1}{2}} W_{\ell-\frac{1}{4}, \frac{3}{4}}(y) & =W_{\ell+\frac{1}{4}, \frac{1}{4}}(y)+\ell W_{\ell-\frac{3}{4}, \frac{1}{4}}(y) .
\end{aligned}
$$

Using these relations, we find that

$$
W_{\ell+\frac{5}{4}, \frac{1}{4}}(y)=-\left(2 \ell+\frac{1}{2}\right) W_{\ell+\frac{1}{4}, \frac{1}{4}}(y)+y W_{\ell+\frac{1}{4}, \frac{1}{4}}(y)-\left(\ell^{2}-\frac{\ell}{2}\right) W_{\ell-\frac{3}{4}, \frac{1}{4}}(y),
$$

and combining this with (3.6) gives (3.5).

Putting these results together with the analogous results for the terms involving the functions $\widetilde{\mathcal{W}}_{\frac{3}{4}}^{2 \ell+\frac{1}{2}}(y)$ gives, by linearity,

$$
\begin{aligned}
R_{2 \ell+\frac{1}{2}}\left(f_{2 \ell+\frac{1}{2}, \chi}(z)\right)= & \sum_{n=-\infty}^{-1} n\left(\ell^{2}+\frac{3 \ell}{2}+\frac{1}{2}\right) a_{2 \ell+\frac{1}{2}}(n) \mathcal{W}_{\frac{3}{4}}^{2 \ell+\frac{5}{2}}(4 \pi n y) e^{2 \pi i n x} \\
+ & \sum_{n=1}^{\infty} n a_{2 \ell+\frac{1}{2}}(n) \mathcal{W}_{\frac{3}{4}}^{2 \ell+\frac{5}{2}}(4 \pi n y) e^{2 \pi i n x} \\
-a_{2 \ell+\frac{1}{2}}(0)(\ell+1)(4 \pi y)^{-\frac{1}{2}-\ell}-\widetilde{a}_{2 \ell+\frac{1}{2}}(0)\left(\ell+\frac{1}{2}\right)(4 \pi y)^{-1-\ell} & \\
- & \sum_{n=-n_{0}}^{-1} n \widetilde{a}_{2 \ell+\frac{1}{2}}(n) \widetilde{\mathcal{W}}_{\frac{3}{4}}^{2 \ell+\frac{5}{2}}(4 \pi n y) e^{2 \pi i n x} \\
& -\sum_{n=1}^{m_{0}} n\left(\ell^{2}+\frac{3 \ell}{2}+\frac{1}{2}\right) \widetilde{a}_{2 \ell+\frac{1}{2}}(n) \widetilde{\mathcal{W}}_{\frac{3}{4}}^{2 \ell+\frac{5}{2}}(4 \pi n y) e^{2 \pi i n x} .
\end{aligned}
$$

From this we conclude that if $r>0$, then

$$
\begin{aligned}
& +\sum_{n=-\infty}^{-1} n^{r} \rho_{r} a_{\frac{1}{2}}(n) \mathcal{W}_{\frac{3}{4}}^{2 r+\frac{1}{2}}(4 \pi n y) e^{2 \pi i n x}+\sum_{n=1}^{\infty} n^{r} a_{\frac{1}{2}}(n) \mathcal{W}_{\frac{3}{4}}^{2 r+\frac{1}{2}}(4 \pi n y) e^{2 \pi i n x} \\
+ & \sum_{n=-n_{0}}^{-1}(-n)^{r} \widetilde{a}_{\frac{1}{2}}(n) \widetilde{\mathcal{W}}_{\frac{3}{4}}^{2 r+\frac{1}{2}}(4 \pi n y) e^{2 \pi i n x}+\sum_{n=1}^{m_{0}}(-n)^{r} \rho_{r} \widetilde{a}_{\frac{1}{2}}(n) \widetilde{\mathcal{W}}_{\frac{3}{4}}^{2 r+\frac{1}{2}}(4 \pi n y) e^{2 \pi i n x}
\end{aligned}
$$


where

$$
\tau_{r}:=\left(-\frac{1}{4}\right)^{r} \frac{(2 r) !}{r !} \quad \text { and } \quad \rho_{r}:=\frac{(2 r) !}{4^{r}} .
$$

We finish by proving the existence of the forms $f_{v, \chi}(z)$. For $1 \leq a \leq b-1$ coprime to $b$ let

$$
\Theta_{\frac{1}{2}, a, b}(z):=\sum_{n \equiv a} q_{(\bmod b)}^{n^{2}}
$$

In [5] the authors construct a harmonic weak Maass form $f_{\frac{3}{2}, a, b}(z)$ of weight $\frac{3}{2}$ on $\Gamma\left(4 b^{2}\right)$ which is the sum of a holomorphic function with algebraic coefficients and a constant multiple of

$$
\int_{-\bar{z}}^{i \infty} \frac{\Theta_{\frac{1}{2}, a, b}(\tau)}{(-i(\tau+z))^{3 / 2}} d \tau
$$

By work of Shimura [17] the function

$$
\Theta_{\frac{1}{2}, \chi}(z):=\sum_{n \in \mathbb{Z}} \chi(n) q^{n^{2}}
$$

is a weight $\frac{1}{2}$ modular form on $\Gamma_{0}\left(4 b^{2}\right)$ with Nebentypus $\chi$. Then

$$
f_{\frac{3}{2}, \chi}(z):=\sum_{a=1}^{b-1} \chi(a) f_{\frac{3}{2}, a, b}(z)
$$

satisfies the hypotheses of Theorem 1.1, and one can check that the holomorphic and nonholomorphic parts of $f_{\frac{3}{2}, \chi}(z)$ have expansions of the form (1.3) and (1.4), respectively (see Proposition 4.1 of 7 for the computation of an expansion of a function like (3.8)). The function $f_{\frac{3}{2}, \chi}^{h}(z)$ is given as a linear combination of basic hypergeometric functions (see [5]).

The case where $v=\frac{1}{2}$ can be dealt with in a similar way (see [4]). Here the corresponding theta function is

$$
\Theta_{\frac{3}{2}, \chi}(z):=\sum_{n \in \mathbb{Z}} \chi(n) n q^{n^{2}} .
$$

Proof of Theorem 1.2, Assume that $w=2 r$ is even (the odd case can be dealt with in an analogous way). As we have seen above, the coefficients of $f_{\frac{1}{2}, \chi}^{h}(z)$ lie in $\mathcal{O}_{K}$ for some number field $K$ and $f_{\frac{1}{2}, \chi}^{n h}(z)$ has the form (1.4). Then our result holds for the harmonic weak Maass form $f_{\frac{1}{2}, \chi}(z)$ by Theorem 1.1 of [13]. By (3.7), for $r>0$ we have that $a_{2 r+\frac{1}{2}}(n)=n^{r} a_{\frac{1}{2}}(n)$ for all $n>0$, and therefore our result holds for the form $f_{w+\frac{1}{2}, \chi}(z)$ in general.

\section{ACKNOWLEDGEMENTS}

The authors wish to thank Ken Ono for helpful discussions during the preparation of this paper and the referee for comments which improved the exposition. 


\section{REFERENCES}

[1] M. Abramowitz and I. A. Stegun, Handbook of mathematical functions, Dover Publications, 1965.

[2] K. Bringmann, Asymptotics for rank partition functions, Trans. Amer. Math. Soc., accepted for publication.

[3] K. Bringmann, On certain congruences for Dyson's ranks, Int. J. Number Theory, accepted for publication.

[4] K. Bringmann, J. Bruinier, A. Folsom and K. Ono, Harmonic Maass forms and Borcherds products, in preparation.

[5] K. Bringmann, A. Folsom and K. Ono, q-series and weight $3 / 2$ Maass forms, submitted for publication.

[6] K. Bringmann and K. Ono, The $f(q)$ mock theta function conjecture and partition ranks, Invent. Math. 165 (2006), pages 243-266. MR2231957 (2007e:11127)

[7] K. Bringmann and K. Ono, Dyson's ranks and Maass forms, Ann. of Math., accepted for publication.

[8] K. Bringmann and K. Ono, Arithmetic properties of coefficients of half-integral weight MaassPoincaré series, Math. Ann. 337 (2007), pages 591-612. MR2274544(2007m:11064)

[9] K. Bringmann and K. Ono, Lifting elliptic cusp forms to Maass forms with an application to partitions, Proc. Nat. Acad. Sci. 104 (2007), no. 10, pages 3725-3731. MR2301875

[10] J. H. Bruinier and J. Funke, On two geometric theta lifts, Duke Math. J. 125 (2004), pages 45-90. MR2097357 (2005m:11089)

[11] J-H. Brunier, P. Jenkins, K. Ono, Hilbert class polynomials and traces of singular moduli, Math. Ann. 334 (2006), pages 373-393. MR 2207703 (2007b:11055)

[12] S. Garthwaite, The coefficients of the $\omega(q)$ mock theta function, Int. J. Number Theory, accepted for publication.

[13] S. Garthwaite and D. Penniston, p-adic properties of Maass forms arising from theta series, Math. Res. Lett., accepted for publication.

[14] F. Hirzebruch and D. Zagier, Intersection numbers of curves on Hilbert modular surfaces and modular forms with Nebentypus, Invent. Math. 36 (1976), pages 57-113. MR0453649 $(56: 11909)$

[15] K. Ono, Distribution of the partition function modulo m, Ann. of Math. 151 (2000), pages 293-307. MR 1745012 (2000k:11115)

[16] S. Ramanujan, The lost notebook and other unpublished papers, Narosa, New Delhi, 1988. MR947735 (89j:01078)

[17] G. Shimura, On modular forms of half integral weight, Ann. of Math. 97 (1973), pages 440481. MR.0332663(48:10989)

School of Mathematics, University of Minnesota, Minneapolis, Minnesota 55455

Current address: Mathematisches Institut, Universität Köln, Weyertal 86-90, 50931 Köln, Germany

E-mail address: kbringma@math.uni-koeln.de

Department of Mathematics, Furman University, Greenville, South Carolina 29613

E-mail address: david.penniston@furman.edu 